\title{
On the reduction of Blackstock's model of thermoviscous compressible flow via Becker's assumption
}

\author{
Rainer Brunnhuber ${ }^{\mathrm{a}}$, P. M. Jordan ${ }^{\mathrm{b}, *}$ \\ ${ }^{a}$ Institut für Mathematik, Universität Klagenfurt Universitätsstraße 65-57, 9020 Klagenfurt am Wörthersee, Austria \\ ${ }^{b}$ Acoustics Div., U.S. Naval Research Laboratory, Stennis Space Ctr., MS 39529, USA
}

\begin{abstract}
Issues regarding the derivation and applicability of the weakly-nonlinear model presented by Brunnhuber and Kaltenbacher in their article [Disc. Cont. Dyn. Sys., Ser. A (DCDS-A) 34 (2014), 4515-4535] are revisited and clarified; in particular, its links to both Blackstock's thermoviscous compressible flow model and Becker's assumption are established.
\end{abstract}

Keywords: Nonlinear acoustics, thermoviscous compressible flow, Becker's assumption

The focus of this Letter is the partial differential equa- 24 tion (PDE)

$$
\begin{aligned}
{\left[\left(\frac{v}{\operatorname{Pr}}\right) \nabla^{2}-\partial_{t}\right](} & \left.\phi_{t t}-c_{0}^{2} \nabla^{2} \phi-\delta \nabla^{2} \phi_{t}\right) \\
& =\partial_{t t}\left[|\nabla \phi|^{2}+\frac{1}{2} c_{0}^{-2}(B / A)\left(\phi_{t}\right)^{2}\right],
\end{aligned}
$$

which Brunnhuber and Kaltenbacher [1] put forth, based in part on Crighton's [2, Eqs. (11), (13)1, as a weakly-nonlinear model of compressible flow. Here, employing a somewhat different notation convention than that of Ref. [1], $\phi=\phi(x, y, z, t)$ is the scalar velocity potential; $c_{0}(>0)$ represents the sound speed in the undisturbed fluid; $v(>0)$ is the kinematic viscosity; $\operatorname{Pr}=v / \kappa$ denotes the Prandtl number, where $\kappa(>0)$ is the thermal diffusivity; $B / A$ is known as the nonlinearity parameter [4]; and $\delta$ is defined in Eq. (5) below.

Specifically, the present authors seek to answer the following question: Given that the derivation presented in Ref. [1] relies on largely heuristic arguments-some of which may not be justified, physically-for what values (if any) of its coefficients is Eq. (1) a realistic mode vis-à-vis finite-amplitude propagation in compressible fluids?

\footnotetext{
${ }^{*}$ Corresponding author.

Email address: pjordan@nrlssc.navy .mil (P. M. Jordan)

${ }^{1}$ We observe that Ref. [2, Eqs. (10)-(13)] are re-statements of PDEs derived by Blackstock [3].

${ }^{2}$ By 'model' we mean a two-equation system involving a PDE for $\phi$ and another from which the rotational part of the velocity vector can be obtained; here, however, the role of the latter is always filled by Eq. 2a below.
}

In the analysis that follows, we derive, what appears to be, a previously unrecognized special case of Blackstock's [3] weakly-nonlinear mode] of thermoviscous compressible flow, one which is to some degree amiable to study by analytical methods; see Refs. [1, 5]. What is perhaps most significant about this special case is the fact that it stems from Becker's assumption, i.e., the assumption that the fluid under consideration is a monatomic perfect gas with $\operatorname{Pr}=3 / 4$; see Becker [6], as well as Morduchow and Libby [7] and Hayes [8].

To this end, we begin by observing that Blackstock actually presents two versions of his model, the first specialized to the case of perfect gases (Ref. [3, §4.1]) and the second applicable to compressible Newtonian fluids in general (Ref. [3, §4.2]). In light of our intent to invoke Becker's assumption, we hereafter limit our focus to the former and re-state, using a slightly modified version of Blackstock's notation, Ref. [3, Eqs. (5b'), (7)], i.e., the system governing propagation in perfect gases:

$$
\begin{gathered}
\boldsymbol{\Psi}_{t}+v \nabla \times(\nabla \times \boldsymbol{\Psi})=0 \\
-\left(v c_{0}^{2} / \operatorname{Pr}\right) \nabla^{4} \phi+v(b+\gamma / \operatorname{Pr}) \nabla^{2} \phi_{t t}+c_{0}^{2} \nabla^{2} \phi_{t}-\phi_{t t t} \\
=\partial_{t}\left[\partial_{t}|\nabla \phi|^{2}+(\gamma-1) \phi_{t} \nabla^{2} \phi\right] .
\end{gathered}
$$

Here, $\boldsymbol{\Psi}=\boldsymbol{\Psi}(x, y, z, t)$, the vector velocity potential, and $\phi$ are defined by

$$
\mathbf{u}=\nabla \phi+\nabla \times \boldsymbol{\Psi}
$$

${ }^{3}$ Strictly speaking, a slight generalization thereof; see Eq. (4) of the present Letter.

1 
where $\mathbf{u}=(u, v, w)$ is the velocity vector; $\nabla^{4}$ repre- 94 sents the bi-Laplacian operator; $\gamma$ denotes the ratio of 95 specific heats, where the specific heats are constants and $\gamma \in(1,5 / 3]$ under the perfect gas assumption [12]; $b=4 / 3+\mu_{\mathrm{B}} / \mu$ is the viscosity number, where $\mu(>0)$ and $\mu_{\mathrm{B}}(\geq 0)$ are the shear and bulk viscosity coefficients, respectively; and we recall that $v$ is the kinematic viscosity coefficient. Also, $c_{0}$ now represents the speed of sound in the undisturbed gas; therefore, here and in what follows it is given by $c_{0}=\sqrt{\gamma P_{0} / \varrho_{0}}$, where the positive constants $P_{0}$ and $\varrho_{0}$ denote the equilibrium state values of the thermodynamic pressure [i.e., $P=$ $P(x, y, z, t)]$ and the mass density [i.e., $\varrho=\varrho(x, y, z, t)],{ }_{103}$ respectively.

Under the approximation scheme set down by Blackstock (Ref. [3, pp. 17-18]), both nonlinear and linear terms whose coefficients are of sufficiently small magnitude were deemed negligible, and thus omitted from the aforementioned governing systems. If, however, we relax this convention so as to allow all linear terms to be retained, while still neglecting those sufficiently small nonlinear ones, Eq. (2b) generalizes to

$$
\begin{aligned}
-\left(b \gamma v^{2} / \operatorname{Pr}\right) \nabla^{4} \phi_{t}- & \left(v c_{0}^{2} / \operatorname{Pr}\right) \nabla^{4} \phi+(v / \operatorname{Pr}) \nabla^{2} \phi_{t t} \\
+\delta & \nabla^{2} \phi_{t t}+c_{0}^{2} \nabla^{2} \phi_{t}-\phi_{t t t} \\
= & \partial_{t}\left[\partial_{t}|\nabla \phi|^{2}+(\gamma-1) \phi_{t} \nabla^{2} \phi\right] .
\end{aligned}
$$

Here, for later use, we have introduced the coefficient

$$
\delta=v[b+(\gamma-1) / \operatorname{Pr}],
$$

which is known as the diffusivity of sound; see, e.g., Pierce [9, §10-8] and Hamilton and Morfey [10, §5]. It should be noted that the first term on the left-hand side (LHS) of Eq. (4) was the only linear term 4 omitted from Ref. [3, Eqs. (7), (12) $\sqrt{5}$ ] under Blackstock's approximation scheme.

Now, on rearranging terms and factoring operators, it ${ }^{125}$ is not difficult to recast Eq. (4) in the form

$$
\begin{aligned}
&-(v / \operatorname{Pr}) \nabla^{2}\left[c_{0}^{2} \nabla^{2} \phi-\phi_{t t}+b \gamma \nu \nabla^{2} \phi_{t}\right] \\
&+\partial_{t}\left[c_{0}^{2} \nabla^{2} \phi-\phi_{t t}+\delta \nabla^{2} \phi_{t}\right] \\
&=\partial_{t}\left[\partial_{t}|\nabla \phi|^{2}+(\gamma-1) \phi_{t} \nabla^{2} \phi\right]
\end{aligned}
$$

\footnotetext{
${ }^{4}$ We observe that this fact, and the fact that Lamb's linear thermoviscous system (Ref. [11, Eqs. (26), (27)]) can be shown to admit the (1D) equivalent of $-\left(b \gamma v^{2} / \operatorname{Pr}\right) \nabla^{4} \phi_{t}$, are at odds with Blackstock's [3, p. 20] assertion that this term 'represents a kind of nonlinear interaction between heat conduction and viscosity.'

${ }^{5}$ Note that the coefficient of the Laplacian on the right-hand side (RHS) of this PDE should read ' $\mathrm{a}_{1} \phi_{t}$ ', where $\mathrm{a}_{1}$ is used in Ref. [3] to denote $B / A$.
}

Clearly, further factorization of the LHS is possible if and only if $\delta=b \gamma v$; i.e., if and only if the equality

$$
b+(\gamma-1) / \operatorname{Pr}=b \gamma,
$$

where we have made use Eq. (5), holds true for physically allowable values of the parameters involved. On solving for $b$, using the fact that $\gamma>1$, we find that this condition can be reduced to $b=1 / \mathrm{Pr}$; however, $b=4 / 3+\mu_{\mathrm{B}} / \mu$, as noted above, and so the condition of equality we seek to satisfy can be re-expressed as

$$
\operatorname{Pr}=\frac{3}{4+3 \mu_{\mathrm{B}} / \mu}
$$

As is well known, 2/3 $\leq \operatorname{Pr}<1$ in the case of perfect gases, to which we have restricted our attention; therefore, we need only consider those gases for which $0 \leq \mu_{\mathrm{B}} / \mu \leq 1 / 6$. A survey of the literature (see, e.g., Refs. [12, Table 1.1] and [13]) reveals that there is only one class of gases whose viscosity ratio falls within, or at an endpoint of, this range of values. That class, of course, is monatomic gases, specifically, gases whose bulk viscosity coefficient is zero [9, p. 550], and of which examples include $\mathrm{Ar}, \mathrm{He}$, and $\mathrm{Ne}$.

On setting $\mu_{\mathrm{B}}=0$, Eq. (8) yields $\mathrm{Pr}=3 / 4$ and the viscosity number becomes $b=4 / 3$. Thus, it follows that $\delta=\delta^{*}$, where $\delta^{*}:=(4 / 3) \gamma v$, and Eq. (6) is reduced to

$$
\begin{aligned}
{\left[\left(\delta^{*} / \gamma\right) \nabla^{2}-\partial_{t}\right] } & \left(\phi_{t t}-c_{0}^{2} \nabla^{2} \phi-\delta^{*} \nabla^{2} \phi_{t}\right) \\
& =\partial_{t}\left[\partial_{t}|\nabla \phi|^{2}+(\gamma-1) \phi_{t} \nabla^{2} \phi\right] .
\end{aligned}
$$

Now using the linear approximation $\nabla^{2} \phi \approx c_{0}^{-2} \phi_{t t}$ to replace, as allowed under the weakly-nonlinear scheme, the product $\phi_{t} \nabla^{2} \phi$ with $\frac{1}{2} c_{0}^{-2} \partial_{t}\left(\phi_{t}\right)^{2}$, Eq. (9) becomes

$$
\begin{array}{r}
{\left[\left(\delta^{*} / \gamma\right) \nabla^{2}-\partial_{t}\right]\left(\phi_{t t}-c_{0}^{2} \nabla^{2} \phi-\delta^{*} \nabla^{2} \phi_{t}\right)} \\
=\partial_{t t}\left[|\nabla \phi|^{2}+\frac{1}{2} c_{0}^{-2}(\gamma-1)\left(\phi_{t}\right)^{2}\right] .
\end{array}
$$

Noting that $B / A=\gamma-1$ in the case of perfect gases (see Ref. [4, p. 27]), its easy to see that Eq. (10) is the special case of Eq. (1) corresponding to a monatomic gas with $\operatorname{Pr}=3 / 4$.

In closing, we call attention to the following: (i) under Becker's assumption, Eq. (4) is, via Eq. (9), reducible to Eq. (10); (ii) Eq. (10) appear to be the only

\footnotetext{
${ }^{6}$ We hedge here, slightly, because it is within the realm of possibility that a gas (or gases) might be found to admit $0<\mu_{\mathrm{B}} / \mu \leq 1 / 6$, and thus satisfy Eq. 8, under certain extreme conditions.
} 
physically justifiable version of Eq. 11); (iii), the range 185 of applicability of Eq. (1) is far more restricted than ${ }^{186}$ that implied in the derivation presented by Brunnhuber ${ }^{187}$ and Kaltenbacher [1], who, it should be noted, actually ${ }_{189}^{188}$ studied the 'Westervelt version' 7 of this PDE; and (iv), 190 Eq. (8) is equivalent to the generalization (i.e., $P r^{\prime \prime}=1,{ }^{191}$ where $\operatorname{Pr}^{\prime \prime}=b \mathrm{Pr}$ ) of Becker's assumption noted on ${ }_{193}^{192}$ Ref. [8, p. 39].

\section{Acknowledgements}

The authors thank Prof. Barbara Kaltenbacher for sharing her insight. The authors also thank Contributing Ed. Prof. Giuseppe Saccomandi for his expeditious handling and able review of the present Letter. R.B. acknowledges the support by the Austrian Science Fund (FWF): P24970 and the Karl Popper Kolleg "ModelingSimulation-Optimization," funded by the Alpen-AdriaUniversität Klagenfurt and by the Carinthian Economic Promotion Fund (KWF). P.M.J. was supported by ONR funding.

\section{References}

[1] R. Brunnhuber, B. Kaltenbacher, Well-posedness and asymptotic behavior of solutions for the Blackstock-CrightonWestervelt equation, Disc. Cont. Dyn. Sys., Ser. A (DCDS-A) 34 (2014), 4515-4535.

[2] D.G. Crighton, Model equations of nonlinear acoustics, Ann. Rev. Fluid Mech. 11 (1979), 11-33.

[3] D.T. Blackstock, Approximate equations governing finiteamplitude sound in thermoviscous fluids, Technical Report GD/E Report GD-1463-52, General Dynamics Corp., Rochester, NY, 1963, Chap. IV.

[4] R.T. Beyer, The parameter $B / A$, in: M.F. Hamilton, D.T. Blackstock (Eds.), Nonlinear Acoustics, Academic Press, San Diego, CA, 1997, Chap. 2.

[5] R. Brunnhuber, Well-posedness and exponential decay of solutions for the Blackstock-Crighton-Kuznetsov equation, J. Math. Anal. Appl. 433 (2016), 1037-1054.

[6] R. Becker, Stoßbwelle und detonation (in German), Z. Phys. 8 (1922), 321-362. [English transl.: Impact waves and detonation, Part I, N.A.C.A. Technical Memo. No. 505 (N.A.C.A., Washington, DC, 1929); Part II, N.A.C.A. Technical Memo. No. 506 (N.A.C.A., Washington, DC, 1929).]

[7] M. Morduchow, P.A. Libby, On a complete solution of the onedimensional flow equations of a viscous, heat-conducting, compressible gas, J. Aero. Sci. 16 (1949), 674-684, and 704.

[8] W.D. Hayes, Gasdynamic Discontinuities, Princeton University Press, Princeton, NJ, 1960, §D,5.

[9] A.D. Pierce, Acoustics: An Introduction to its Physical Principles and Applications, Acoustical Society of America, Woodbury, NY, 1989.

\footnotetext{
${ }^{7}$ By which we mean, in the case of Eq. 10, further approximating the RHS to reduce it to $\frac{1}{2} c_{0}^{-2}(\gamma+1) \partial_{t t}\left[\left(\phi_{t}\right)^{2}\right]$.
}

[10] M.F. Hamilton, C.L. Morfey, Model equations, in: M.F. Hamilton, D.T. Blackstock (Eds.), Nonlinear Acoustics, Academic Press, San Diego, CA, 1997, Chap. 3.

[11] H. Lamb, Hydrodynamics, 6th edn., Dover, New York, 1945, Art. 360 .

[12] P.A. Thompson, Compressible-Fluid Dynamics, McGraw-Hill, New York, 1972.

[13] M.S. Cramer, Numerical estimates for the bulk viscosity of ideal gases, Phys. Fluids 24 (2012), 066102. 